\title{
MHSalud
}

MHSalud

ISSN: 1659-097X

revistamhsalud@una.cr

Universidad Nacional

Costa Rica

\section{Procesos de percepción auditiva y aprendizaje motor. Revisión bibliográfica}

Montoya, Olga Lucia; Serna, Gladys Iliana; Martínez, Oswal

Procesos de percepción auditiva y aprendizaje motor. Revisión bibliográfica

MHSalud, vol. 19, núm. 1, 2022

Universidad Nacional, Costa Rica

Disponible en: https://www.redalyc.org/articulo.oa? $\mathrm{id}=237068652006$

DOI: https://doi.org/10.15359/mhs.19-1.7

\section{(c) (1) (8)}

Esta obra está bajo una Licencia Creative Commons Atribución-NoComercial-SinDerivar 3.0 Internacional, 
MHSALUD, ISSN: 1659-097X, 19(1), ENERo-JuNIO, 2022, PP 1-10

Montoya, Serna, Martínez

\section{Procesos de percepción auditiva y aprendizaje motor. Revisión bibliográfica}

Auditory perception processes and motor learning. Bibliographic Review

Processos de percepção auditiva e aprendizagem motora. Revisão bibliográfica

Olga Lucia Montoya

Escuela Colombiana de Rehabilitación, Escuela de

Fisioterapia. Grupo de Investigación Capacidades

DOI: https://doi.org/10.15359/mhs.19-1.7

Humanas, Salud e Inclusión, Colombia

olga.montoya@ecr.edu.co

(iD https://orcid.org/0000-0002-9540-1581

Gladys Iliana Serna

Universidad de Guadalajara, México

gladys.serna98@gmail.com

(D) https://orcid.org/0000-0001-6336-1063

Oswal Martínez

Escuela Colombiana de Rehabilitación, Escuela de

Fonoaudiología. Grupo de Investigación Capacidades

Humanas, Salud e Inclusión, Colombia

oswal.martinez@ecr.edu.co

(D) https://orcid.org/0000-0002-0266-5116

Redalyc: https://www.redalyc.org/articulo.oa?

id $=237068652006$

Recepción: 15 Abril 2021

Aprobación: 17 Agosto 2021

\section{Resumen:}

Introducción: A nivel neurofisiológico el cerebelo, los ganglios basales y el sistema límbico son importantes en la coordinación y memoria del movimiento. Objetivo: Comprender los procesos que intervienen en la relación sensoperceptiva de la audición y el aprendizaje motor es una motivación permanente de diferentes disciplinas. Método: Se presenta una revisión documental que tuvo por objetivo analizar la relación de la percepción auditiva y el aprendizaje motor utilizando análisis de contenido desde las perspectivas de audiología, medicina y neurorrehabilitación. Las palabras clave y combinaciones que se tuvieron en cuenta fueron: percepción auditiva, aprendizaje, equilibrio, coordinación y las combinaciones audición-aprendizaje, audición-equilibrio, y audición-coordinación. Se utilizaron las bases de datos y metabuscadores Pubmed, Medscape, Trip, ScienceDirect, EBSCOhots, Pedro, Scielo, y Lilacs. Bibliotecas virtuales como SINAB, Cochrane, Universidad de Málaga, UsNational Library of Medicine, y National Institutes of Health. Se seleccionaron 22 artículos que cumplieron con los criterios de inclusión. Resultados: Se encontró relación entre la percepción auditiva y el aprendizaje motor en la comunicación de la información sensorial auditiva y motora a nivel del procesamiento en el cerebelo y ganglios, que es una parte fundamental en la retención y transferencia motriz. Conclusión: En el proceso del aprendizaje motor que involucra la experiencia del movimiento, proponemos la participación de la audición, mediante integrar las señales percibidas-visuales, auditivas, motrices y vestibulares- que se concretan en mejorar el aprendizaje, haciéndolo más eficaz y generando una memoria más duradera.

Palabras ClaVE: Percepción auditiva, aprendizaje, equilibrio postural.

\section{ABstRACT:}

Introduction: At the neurophysiological level the cerebellum, the basal ganglia and the limbic system are important in the coordination and memory of movement. Objective: To understand the processes involved in the sensoperceptive relationship of hearing and motor learning is a permanent motivation of different disciplines. Method: A documentary review is presented that aimed to analyze the relationship of auditory perception and motor learning using content analysis from the perspectives of audiology, medicine and neurorehabilitation. The key words and combinations taken into account were: hearing perception, learning, balance, coordination, and combinations hearing-learning, hearing-balance, and hearing-coordination. The databases and metasearchers Pubmed, Medscape, Trip, ScienceDirect, EBSCOhots, Pedro, Scielo, and Lilacs were used. Virtual libraries such as SINAB, Cochrane, University of Malaga, UsNational Library of Medicine, and National Institutes of Health. We selected 
22 articles that met the inclusion criteria. Results: A relationship was found between auditory perception and motor learning in the communication of auditory and motor sensory information at the level of processing in the cerebellum and basal ganglia, being a fundamental part of the retention and motor transfer. Conclusion: In the process of motor learning that involves the experience of movement, we propose the participation of hearing, by integrating the perceived signals -visual, auditory, motor and vestibular- that are concretized to improve learning, making it more effective, generating a more lasting memory.

KEYWORDS: auditory perception, learning, motor skills, postural balance.

\section{Resumo:}

Introdução: A nível neurofisiológico, o cerebelo, os gânglios basais e o sistema límbico são importantes na coordenação e memória do movimento. Objetivo: Compreender os processos que implicam na relação sensorial-perceptual entre audição e aprendizagem motora como uma motivação permanente para diferentes disciplinas. Método: É apresentada uma revisão documental que visa analisar a relação entre a percepção auditiva e a aprendizagem motora utilizando a análise de conteúdo a partir das perspectivas da audiologia, medicina e neuro reabilitação. As palavras-chave e as combinações consideradas foram: percepção auditiva, aprendizagem, equilíbrio, coordenação e as combinações audição-aprendizagem, audição-equilíbrio e audição-coordenação. Foram utilizadas as bases de dados e os motores de metabusca Pubmed, Medscape, Trip, ScienceDirect, EBSCOhots, Pedro, Scielo, e Lilacs. Bibliotecas virtuais como SINAB, Cochrane, Universidade de Málaga, UsNational Library of Medicine, e National Institutes of Health. Foram selecionados 22 artigos que cumpriam os critérios de inclusão. Resultados: Foi encontrada uma relação entre percepção auditiva e aprendizagem motora na comunicação da informação sensorial auditiva e motora à nível do processamento no cerebelo e nos gânglios, que é uma parte fundamental da retenção e transferência motora. Conclusão: No processo de aprendizagem motora que envolve a experiência do movimento, propomos a participação da audição, integrando os sinais percebidos - visuais, auditivos, motores e vestibulares - que resultam na melhoria da aprendizagem, tornando-a mais eficaz e gerando uma memória mais duradoura.

Palavras-Chave: Percepção auditiva, aprendizagem, equilíbrio postural.

\section{INTRODUCCIÓN}

Las autoras y el autor del presente artículo declaran que todos han tenido un grado de contribución sustancial para la elaboración del documento, ya sea desde su conceptualización y diseño, el análisis o interpretación de datos, la redacción y revisión crítica o el control de calidad para su publicación.

Comprender el proceso auditivo y la codificación de las ondas sonoras es complejo. Primero el sonido penetra por el conducto auditivo externo y hace vibrar una membrana conocida como tímpano; este se encuentra en íntima relación con el sistema de huesecillos del oído medio, que amplifican las señales recibidas y las transmiten a la cóclea (Sánchez, 2014). El órgano de Corti, que se encuentra en la cóclea, tiene la función de traducir los estímulos sonoros que llegan a través de los líquidos endolinfáticos y perilinfáticos en impulsos bioeléctricos a través de la despolarización de las células ciliadas internas. Estos impulsos se trasmiten por los axones de las células especializadas que forman el núcleo coclear y viajan hasta el tallo cerebral, en donde se transmiten por los tubérculos cuadrigéminos inferiores, para posteriormente pasar a los cuerpos geniculados mediales (Sánchez, 2014). Cada una de estas estructuras contribuye con el procesamiento del sonido, en términos de interpretación del tono, la intensidad, la duración, entre otros aspectos. Finalmente, los impulsos llegan al lóbulo temporal, específicamente a las áreas 41 y 42 de Brodmann, en donde se realiza un análisis acústico con más profundidad, se realiza la interpretación y se le da significado al sonido (Kramer \& Brown, 2021). Lo anterior describe los procesos sensoriales de la vía auditiva antes de iniciar la comprensión de los procesos perceptivos, los cuales son de interés para el presente estudio.

El movimiento corporal humano ha sido comprendido desde una actividad refleja y automática hasta asumirlo como un proceso complejo y dinámico que lleva implícitas experiencias de vida y emociones; en estas últimas comprensiones el aprendizaje motor ha sido importante para los análisis de patrones motores debido a que las zonas cerebrales que intervienen en la planeación, programación y ejecución del movimiento también influyen en los procesos de aprendizaje. 
El aprendizaje motor, al igual que otros aprendizajes, consta de diversas fases en donde la información sensorial, la memoria y la motivación interactúan para conseguir un resultado óptimo; su comprensión ha evolucionado desde sus inicios en 1820 y se le ha estudiado en diferentes contextos terapéuticos, recibiendo aportes multidisciplinarios desde medicina, neurofisiología, neuropsicología y neurorrehabilitación. Inicialmente fue estudiado por psicólogos y médicos interesados en indagar cómo una persona aprendía a moverse; para entonces, la observación y la experimentación fueron el método para analizar estos procesos (Batalla, 2005).

Posteriormente, con la Segunda Guerra Mundial, otras disciplinas interesadas en recuperar el movimiento corporal humano y las habilidades motoras de las personas empezaron a estudiarlo desde un componente biomecánico, comprendiendo cómo se planea y se ejecuta el movimiento para así lograr recuperarlo. Aquí, el concepto de control motor es fundamental para sus análisis.

Desde 1971 hasta la actualidad, se ha desarrollado un periodo de estudio de los procesos del aprendizaje motor teniendo en cuenta la percepción motriz y los componentes que influyen en ella. El aprendizaje motor se puede comprender como las experiencias motrices que una persona adquiere por diferentes momentos de su vida a través de la práctica mental, práctica física, retención y trasferencia motriz (Pérez et al., 2020).

A nivel neurofisiológico, el cerebelo, los ganglios basales y el sistema límbico son los encargados del procesamiento perceptivo motriz. La motivación y la memoria (explícita-implícita) logran generar después de los procesamientos sensoriales, incluyendo las emociones y los sentimientos, huellas motrices que quedan grabadas, disponibles para ser evocadas en el momento que se requiera. Estas memorias motrices hacen parte de la corporalidad y del comportamiento motor (Mena de la Cruz, 2018).

En las últimas décadas ha cobrado importancia saber cómo los procesos perceptivos influyen en el movimiento corporal humano. Con base en esta inquietud surgen investigaciones que revolucionan la neurociencia, al proveer evidencia de actividad en la corteza motora cada vez que se realiza una acción o se observa a otra persona realizarla, proponiendo la conexión entre la vía sensorial y la motora con las llamadas "neuronas espejo" (Rizzolatti et al., 1996, p. 139).

En la ejecución de un movimiento puede estar implicada la vía auditiva, encargada de codificar el lenguaje hablado, sonidos o ambos. La información acústica interviene en los desempeños motores, influye en los mecanismos del control postural y en el patrón motor de la marcha, evidenciándose en los primeros dos años de vida donde se hace mayor integración sensorial y se fortalecen las conductas motrices que dan paso a los patrones locomotores, manipulativos y de estabilidad (Suárez y Ferreira, 2019).

Un experimento realizado con aves mostró que los procesos de vocalización solo pueden aprenderse en un contexto social, ya que es necesario integrar la percepción auditiva al movimiento bucal y contar con una retroalimentación sensorial para realizar la tarea con éxito (Brainard y Doupe, 2002, p. 355).

Desde el deporte se han aproximado a comprender cómo se puede utilizar la sonificación como una herramienta para mediar la información a través del sistema auditivo, y de este modo apoyar los procesos de control motor y aprendizaje. Se pueden abordar las capacidades específicas del sistema auditivo para influir en características específicas del mecanismo del control postural y del equilibrio. Sugieren la importancia de estudios que analicen neurofisiológicamente la integración sensorial de los procesos de percepción auditiva y el impacto en los patrones motores (Effenberg, 2005, p. 56). Se han realizado diversos estudios que demuestran la importancia de la retroalimentación aumentada donde estímulos sonoros y visuales ayudan a corregir errores de rendimiento durante la ejecución de patrones de movimiento; sin embargo, las características perceptivas de estas integraciones sensoriales aún están en estudio (Ronsse et al., 2010, p. 1285).

La influencia de los sonidos generados naturalmente en la ejecución de un movimiento también ha sido foco de interés al mejorar el rendimiento de los atletas, ya sea porque proveen información sobre el entorno o porque ayudan a anticipar movimientos. Por otro lado, la privación de esta retroalimentación obstaculiza el 
desempeño de algunos atletas, como tenistas y corredores, así como en el aprendizaje de secuencias manuales (Kennel et al., 2015; Agostini et al., 2004).

Existen diversos reportes que asocian la hipoacusia con un mayor riesgo de caída, dado que hay una correlación lineal entre la gravedad de la misma y el riesgo, así como estudios que demuestran que mientras más referencias auditivas tenga una persona sana su estabilidad postural es mayor (Lin y Ferrucci, 2012; Viljanen et al., 2009).

Un estudio realizado en Alemania mostró incremento en la actividad contralateral de la corteza motora en pianistas al escuchar una pieza musical, cuyas regiones coincidían con las áreas asociadas al movimiento de los respectivos dedos utilizados al practicar por sí mismos la pieza (Haueisen \& Knösche, 2001).

Realizar mapas auditivos visuales es recomendable para favorecer los procesos sensoriales y perceptivos en relación con la audición y el movimiento; esto permite que la persona identifique los estímulos sonoros y sus características en relación con su cuerpo y movimiento cuando está realizando un patrón motor, lo que le permite una retroalimentación a la persona para identificar en relación al sonido su respuesta motora y realizar ajustes posturales, además de identificar si la contaminación auditiva influye en la capacidad de realizar ajustes motrices (Gandemer et al., 2017).

Por lo anterior se plantea la siguiente pregunta: ¿Cuál es la relación entre la percepción auditiva y el aprendizaje motor?

\section{MÉTODo}

Se presenta una revisión documental de literatura científica que tuvo por objetivo indagar sobre la relación de la percepción auditiva y el aprendizaje motor. Las palabras claves y combinaciones que se tuvieron en cuenta fueron: percepción auditiva, aprendizaje, equilibrio, coordinación y las combinaciones audición-aprendizaje, audición-equilibrio, y audicióncoordinación. Se utilizaron las bases de datos y metabuscadores Pubmed, Medscape, Trip, ScienceDirect, EBSCOhots, Pedro, Scielo, y Lilacs. Bibliotecas virtuales como SINAB, Cochrane, Universidad de Málaga, UsNational Library of Medicine, y National Institutes of Health.

Como criterios de inclusión se tuvieron en cuenta artículos publicados en español e inglés sin límite de año de publicación que tuvieran en resultados descripción de procesos perceptivos de la audición y aprendizaje motor. Se excluyeron artículos que asumían el aprendizaje motor desde una perspectiva mecanicista del movimiento corporal humano sin tener en cuenta los procesos perceptivos y de integración sensorial. De 53 artículos encontrados se seleccionaron 22 que cumplieron con los criterios de inclusión. Se realizó un análisis de contenido desde las perspectivas disciplinares de medicina, neurorrehabilitación y audiología a través de una matriz de análisis de datos y posterior discusión entre las disciplinas. Una vez realizado este análisis, se procedió a darle un orden a las categorías encontradas para una mayor comprensión del lector; se inicia explicando los componentes neurofisiológicos de los procesos perceptivos de la audición y aprendizaje motor, continuando con los procesos de retroalimentación y finalmente se explican las relaciones con los componentes de la percepción auditiva, control y aprendizaje motor.

\section{Resultados}

Neurofisiología de la percepción auditiva y el aprendizaje motor

Se ha estudiado la existencia de neuronas espejo auditivas al activarse un circuito temporo-parietopremotor hemisférico izquierdo y de la corteza premotora izquierda (Gazzola et al., 2006; Schachner, 2010). Esto indica que el sistema límbico está en conexión con las zonas de aprendizaje motor como el cerebelo y los ganglios basales. 
Estudios en músicos demostraron activación de la corteza motora correspondiente al movimiento de las manos al escuchar una pieza bien ejecutada (Lahav et al., 2007; Haueise y Knösche, 2001), lo que apoya la teoría de una red de neuronas espejo auditivas que conectan la percepción auditiva con la corteza motora. Es importante mencionar que, en estos estudios, el estímulo solo se desencadenó en músicos.

En 1959, el neuropsicólogo Donald Hebb propuso la teoría de la "asamblea celular", actualmente conocida como la teoría de Hebb o hebbiana del aprendizaje, que puede resumirse en su frase célebre "Neurons that fire together, wire together" o, traducida al español, "Las neuronas que se activan juntas refuerzan su conexión". Esta teoría habla de cómo se consolida el conocimiento con base a las sinapsis o circuitos neuroanatómicos preexistentes y cómo la repetición de una acción tiene gran peso para generar memoria a largo plazo (Brown, 2020). Los músicos crean redes neuronales con la activación simultánea de la corteza auditiva y la corteza motora al realizar movimientos con sus manos para producir sonido.

La observación de movimientos con sonido congruente comparado a sonido incongruente (retrasado milésimas de segundo) potenció la activación de áreas motoras como el tálamo y el cerebelo, al incrementar el reclutamiento de ganglios basales y al amplificar la actividad neuronal de la red frontocortical y la región temporal superior (Schmitz et al., 2013). Esta última ha sido identificada como un centro integrador multisensorial: visual, auditivo y motor; se advierte cómo la percepción auditiva es compleja y conlleva la transformación de las señales recibidas en la vía auditiva para lograr la codificación del estímulo espectrotemporal más allá del encéfalo (Atencio et al., 2012).

El cerebelo interactúa con los ganglios basales para darle características específicas de ritmo y espacialidad al movimiento corporal humano. Estudios previos en pacientes con daño cerebelar describen el papel del cerebelo y del sistema córtico-estrío-tálamo-cortical en la percepción del tiempo (Meck et al., 2008). Se propone recientemente un modelo unificador que conecta ambas vías para la representación contextual del tiempo, donde se hace referencia a aferencias auditivas del núcleo coclear hacia la oliva inferior, que lleva información al tálamo y al cerebelo, lo que permite contextualizar en el ambiente la representación del tiempo. Es así que la perturbación de los estímulos externos, como la hipoacusia, podría ocasionar una descoordinación con el medio externo, llevando a alteraciones motoras y del equilibrio (Teki et al., 2012).

La estructura y función del fascículo arqueado siguen siendo controversiales. Se trata de un tracto de materia blanca que conecta la corteza temporal lateral con la corteza frontal (Dick y Tremblay, 2012), que tiene importancia en la comunicación al conectar el área de Broca y el área de Wernicke. Existe evidencia de conexión entre la vía auditiva y la corteza motora primaria a través de proyecciones del fascículo arqueado, que se han propuesto como base para la producción del lenguaje; sin embargo, no se ha investigado si podrían tener relación con el equilibrio o la integración motora-sensorial (Fernández-Miranda et al., 2015).

Los procesos perceptivos de la audición, al estar relacionados directamente con el cerebelo, influyen en las funciones cognitivas de las praxias, las cuales permiten saber dónde estamos en relación con eventos y objetos importantes en nuestro entorno activando los ganglios basales, que son los encargados de dar características específicas de movimiento y retroalimentación de los patrones motores perfeccionando ritmo y velocidad y rastreando nuestra posición cambiante dinámicamente en el espacio y el tiempo. La integración de información auditiva y motora impacta la seguridad de las personas en movimientos complejos como caminar o correr; es por esto que algunos estudios han indagado sobre el riesgo de que personas mayores con pérdida auditiva vayan a caer más allá de las alteraciones de los mecanismos vestibulares, y les han dado importancia a los procesos perceptivos (Campos et al., 2018).

\section{Retroalimentación auditiva y control motor}

La retroalimentación auditiva concurrente se ha aplicado con éxito en el aprendizaje motor. En comparación con la retroalimentación visual, la auditiva puede dificultar el procesamiento de otras aferencias sensoriales en menor medida y, por lo tanto, aún podría usarse para calibrar el programa motor como información visual 
escasa. El éxito de la retroalimentación auditiva también puede originarse en el hecho de que la mayoría de los estudios sobre alarmas auditivas y sonificación de movimiento han investigado tareas repetitivas rápidas. Dichas tareas limitan las correcciones de movimiento en línea, es decir, las correcciones de errores irrelevantes causados por el ruido sensorial-motor; en cambio, la información auditiva apoya el control de avance (Effenberg, 2005).

Las habilidades motoras deben incluirse como parte de la evaluación clínica de rutina, así como los programas educativos en niños con discapacidad auditiva. Según lo declarado por Vidranski y Farkaš (2015, p. 173), si a estos niños se les brindan oportunidades apropiadas para practicar, pueden adquirir habilidades motoras fundamentales en la misma secuencia y aproximadamente al mismo ritmo que sus compañeros sin discapacidad. Para minimizar los efectos adversos en el desarrollo normal de los trastornos de la audición, es crucial realizar exámenes de detección e intervenciones apropiadas de déficit de equilibrio y motores que permitan la detección temprana de estas disfunciones, que a menudo no se notan o se subestiman. Puede ser importante a la hora de pensar en promover la actividad física en niños con problemas auditivos a través de la actividad física tendiente a mejorar el bienestar mental y físico, lo que influye en los procesos de memoria y atención que mejoran el desempeño académico (Chacón-Cuberos et al., 2020, p. 8).

\section{Percepción auditiva y aprendizaje motor}

La percepción motriz entendida como el proceso donde se integra la información sensorial en diferentes zonas cerebrales y le da una interpretación y significado para transformarlo en un movimiento corporal visible, incorpora procesamientos de memoria auditiva relacionados con información sensorial de todo tipo que se conecta con zonas de planeación, programación y codificación del movimiento, como lo son los ganglios basales, el cerebelo y el sistema límbico (Pérez et al., 2020). Esto es importante comprenderlo pues en el proceso de aprendizaje motor se debe tener en cuenta la integración sensorial y no solo comandos verbales y visuales; es decir, se deben considerar los diferentes tipos de memorias sensitivas para lograr evocar y guardar movimientos corporales y así lograr hacer retroalimentación motriz.

La importancia de la relación de los procesos de percepción auditiva y motriz ha sido estudiada dando como resultado que, al combinar estímulos sensoriales de sonido con el movimiento, se genera memoria a largo plazo de lo aprendido. Cada vez que nos movemos, hablamos o tocamos instrumentos musicales, nuestras acciones generan una entrada sensorial auditiva. Las consecuencias sensoriales de nuestras acciones se cree que se predicen a través de la integración sensoriomotriz, que implica vínculos anatómicos y funcionales entre las regiones auditivas y del cerebro motor. Las conexiones fisiológicas están relativamente bien establecidas, pero se sabe menos sobre cómo afecta la integración sensorial (Myers et al., 2020)

Los sonidos que se han producido con el propio sistema motor se recuerdan mejor que los sonidos que solo se han percibido, lo que sugiere un papel de la información motora en la memoria para la estímulo auditivo. Los resultados de las interacciones auditivo-motoras contribuyen a la memoria, y esto es atribuido a la experiencia de producción y apoyo a la predicción motora (Brown y Palmer, 2012).

\section{CONCLUSIONES Y RECOMENDACIONES FUTURAS}

La relación de la vía auditiva sensorial con la vía motora desde un análisis neurofisiológico genera una base teórica para la investigación futura y el desarrollo de nuevos abordajes para el aprendizaje motor que pueden ser utilizados en los ámbitos clínico y deportivo.

En el proceso del aprendizaje motor que involucra la visualización y realización propia del movimiento, proponemos la participación de la audición, mediante la integración de las señales percibidas -visuales, auditivas, motrices y vestibulares- que se concretan en mejorar el aprendizaje, haciéndolo más eficaz, así 
como generando una memoria más duradera. Esto puede justificarse desde los componentes neurológicos que intervienen en la conciencia corporal: al involucrar procesos auditivos y motores en el aprendizaje de movimientos en las fases de práctica y retención motriz, se estimulan las zonas cerebrales de integración sensorial y, a su vez, influye directamente en el sistema límbico, cerebelo y ganglios basales, lo que se traduce en un movimiento que se guarda con motivación y con características de ejecución de calidad de movimiento (Skjaerven et al., 2018, p. 86).

Es de interés que este medio sensorial no dificulte ni entorpezca la recepción sensorial de otras índoles. Las señales recibidas se incorporan a la memoria auditiva por medio del sistema límbico, enviándolas a zonas del cerebro encargadas de la planeación y ejecución del movimiento - ganglios basales, médula, fascículo arqueado y cerebelo- para darle un sentido espacial, así como temporal, a la acción realizada, convirtiéndola en más precisa y eficaz (Sigrist et al., 2013; Buchanan y Wang, 2012).

Se sugiere la evaluación auditiva a la par de motora en las fases iniciales de retención motriz para los entrenamientos de patrones motores, con base en los posibles efectos sinérgicos que contribuirían a mejorar la calidad d el movimiento. La visión de audiología, neurorrehabilitación y medicina fue importante para decodificar la información, generando un resultado holístico que se puede interpretar desde múltiples perspectivas.

El uso de la sonificación se ha asociado a mejorar el rendimiento y eficiencia del proceso de aprendizaje motor en deportistas, abriendo oportunidades de investigación y desarrollo tecnológico en este campo donde es importante incluir procesos que favorezcan las praxias desde los componentes perceptivos. Una de las opciones es la imaginería motora.

El abordaje multidisciplinar para la comprensión de los procesos perceptivos del aprendizaje motor se recomienda a la hora de estructurar programas de entrenamiento y rehabilitación, ya que permiten abordajes integrales que tengan en cuenta no solo los procesos de ejecución del movimiento como respuesta final sino procesos que se dan en la planeación y programación motriz.

\section{Agradecimientos}

Este trabajo se ha realizado gracias al programa de pasantías de investigaciones internacionales Red Delfín y a la Escuela Colombiana de Rehabilitación.

\section{DeClaración de CONTRIBUCión DE PERSONAS AUTORAS}

Las autoras y el autor del presente artículo declaran que todos han tenido un grado de contribución sustancial para la elaboración del documento, ya sea desde su conceptualización y diseño, el análisis o interpretación de datos, la redacción y revisión crítica o el control de calidad para su publicación.

\section{REFERENCIAS}

Agostini, T., Righi, G., Galmonte, A. \& Bruno, P. (2004). The relevance of auditory information in optimizing hammer throwers performance. Biomechanics and Sports, 473, 67-74. https://doi.org/10.1007/978-3-7091-2760-5_9

Atencio, C. A., Sharpee, T. O. \& Schreiner, C. E. (2012). Receptive field dimensionality increases from the auditory midbrain to cortex. Journal of Neurophysiology, 107(10), 2594-2603. https://doi.org/10.1152/jn.01025.2011

Batalla Flores, A. (2005). Retroalimentación y aprendizaje motor: influencia de las acciones realizadas de forma previa a la recepción del conocimiento de los resultados en el aprendizaje y la retención de habilidades motrices. Dipòsit Digital de la Universitat de Barcelona. http://hdl.handle.net/2445/43053 
Brainard, M. S. \& Doupe, A. J. (2002). What songbirds teach us about learning. Nature, 417(6886), 351-358. https ://doi.org/10.1038/417351a

Brown, R. E. (2020). Donald O. Hebb and the Organization of Behavior: 17 years in the writing. Molecular Brain, 13(1), 55. https://doi.org/10.1186/s13041-020-00567-8

Brown, R. M. \& Palmer, C. (2012). Auditory-motor learning influences auditory memory for music. Memory \& Cognition, 40(4), 567-578. https://doi.org/10.3758/s13421-011-0177-x

Buchanan, J. J. \& Wang, C. (2012). Overcoming the guidance effect in motor skill learning: feedback all the time can be beneficial. Experimental Brain Research, 219(2), 305-320. https://doi.org/10.1007/s00221-012-3092-x

Campos, J., Ramkhalawansingh, R. \& Pichora-Fuller, M. K. (2018). Hearing, self-motion perception, mobility, and aging. Hearing Research, 369, 42-55. https://doi.org/10.1016/j.heares.2018.03.025

Chacón-Cuberos, R., Zurita-Ortega, F., Ramírez-Granizo, I. \& Castro-Sánchez, M. (2020). Physical Activity and Academic Performance in Children and Preadolescents: A Systematic Review. Apunts. Educación Física y Deportes, (139), 1-9. https://doi.org/10.5672/apunts.2014-0983.es.(2020/1).139.01

Dick, A. \& Tremblay, P. (2012). Beyond the arcuate fasciculus: consensus and controversy in the connectional anatomy of language. Brain, 135(12), 3529-3550. https://doi.org/10.1093/brain/aws222

Effenberg, A. O. (2005). Movement sonification: effects on perception and action. IEEE Multimedia, 12(2), 53-59. https://doi.org/10.1109/mmul.2005.31

Fernández-Miranda, J. C., Wang, Y., Pathak, S., Stefaneau, L., Verstynen, T. \& Yeh, F. C. (2015). Asymmetry, connectivity, and segmentation of the arcuate fascicle in the human brain. Brain Structure \& Function, 220(3), 1665-1680. https://doi.org/10.1007/s00429-014-0751-7

Gandemer, L., Parseihian, G., Kronland-Martinet, R. \& Bourdin, C. (2017). Spatial cues provided by sound improve postural stabilization: evidence of a spatial auditory map? Frontiers in Neuroscience, 11, 1-11. https://doi.org/1 $0.3389 /$ fnins.2017.00357

Gazzola, V., Aziz-Zadeh, L. \& Keysers, C. (2006). Empathy and the somatotopic auditory mirror system in humans. Current Biology, 16(18), 1824-1829. https://doi.org/10.1016/j.cub.2006.07.072

Haueisen, J. \& Knösche, T. R. (2001). Involuntary Motor Activity in Pianists Evoked by Music Perception. Journal of Cognitive Neuroscience, 13(6), 786-792. https://doi.org/10.1162/08989290152541449

Kramer, S. \& Brown, D. K. (2021). Audiología: Ciencia a la práctica (4.. ed.). Plural Publishing, Inc.

Kennel, C., Streese, L., Pizzera, A., Justen, C., Hohmann, T. \& Raab, M. (2015). Auditory reafferences: the influence of the real-time feedback on movement control. Frontiers in Psychology, 6, 1-6. https://doi.org/10.3389/fpsyg .2015 .00069

Lahav, A., Saltzman, E. \& Schlaug, G. (2007). Action representation of sound: audio motor recognition network while listening to newly acquired actions. Journal of Neuroscience, 27(2), 308-314. https://doi.org/10.1523/JNEUR OSCI.4822-06.2007

Lin, F. R. \& Ferrucci, L. (2012). Hearing loss and falls among older adults in the United States. Archives of Internal Medicine, 172(4), 369-371. https://doi.org/10.1001/archinternmed.2011.728

Meck, W. H., Penney, T. B. \& Pouthas, V. (2008). Cortico-striatal representation of time in animals and humans. Current Opinion in Neurobiology, 18(2), 145-152. https://doi.org/10.1016/j.conb.2008.08.002

Mena de la Cruz, A. C. (2018). Aproximación teórica de la intervención de fisioterapia en neurorrehabilitación desde el aprendizaje motor en pacientes con evento cerebrovascular. Movimiento Cientifico, 11(2), 73-80. https://do i.org/10.33881/2011-7191.mct.11204

Myers, J. C., Mock, J. R. \& Golob, E. J. (2020). Sensorimotor integration can enhance auditory perception. Scientific Reports, 10(1), 1-13. https://doi.org/10.1038/s41598-020-58447-z

Pérez, C., Montoya, O. L. \& Henao, L. M. (2020). Aprendizaje motor y realidad virtual. En M. A. Cubillo León (Comp.), Avances cientificos y nuevas tecnologias en neurorrehabilitación transdisciplinaria (pp. 119-141). CMUCH. https://bit.ly/35LTYMW 
Rizzolatti, G., Fadiga, L., Gallese, V. y Fogassi, L. (1996). Premotor cortex and the recognition of motor actions. Cognitive Brain Research, 3(2), 131-141. https://doi.org/10.1016/0926-6410(95)00038-0

Ronsse, R., Puttemans, V., Coxon, J. P., Goble, D. J., Wagemans, J., Wenderoth, N. \& Swinnen, S. P. (2010). Motor learning with augmented feedback: modality-dependent behavioral and neural consequences. Cerebral Cortex, 21(6), 1283-1294. https://doi.org/10.1093/cercor/bhq209

Sánchez, E. (2014). Fisiología auditiva. En J. Pérez \& E. Gil (Eds.), Libro virtual de formación en ORL (1.. ed., Vol. 1, pp. 1-19). Sociedad Española de Otorrinolaringología y Patología Cérvico-Facial. https://booksmedicos.org/li bro-virtual-de-formacion-en-otorrinolaringologia-seorl/

Schachner, A. (2010). Auditory-motor entrainment in vocal mimicking species. Communicative \& Integrative Biology, 3(3), 290-293. https://doi.org/10.4161/cib.3.3.11708

Schmitz, G., Mohammadi, B., Hammer, A., Heldmann, M., Samii, A., Münte, T. F. \& Effenberg, A. O. (2013). Observation of sonified movements engages a basal ganglia frontocortical network. BMC Neuroscience, 14(32). https://doi.org/10.1186/1471-2202-14-32

Sigrist, R., Rauter, G., Riener, R. \& Wolf, P. (2013). Augmented visual, auditory, haptic, and multimodal feedback in motor learning: A review. Psychonomic Bulletin \& Review, 20, 21-53. https://doi.org/10.3758/s13423-012 $-0333-8$

Skjaerven, L. H., Mattsson, M., Catalán-Matamoros, D., Parker, A., Gard, G. \& Gyllensten, A. L. (2018). Consensus on core phenomena and statements describing Basic Body Awareness Therapy within the movement awareness domain in physiotherapy. Physiotherapy Theory and Practice, 35(1), 80-93. https://doi.org/10.1080/09593985 .2018 .1434578

Suárez, H. \& Ferreira, E. (2019). Rol de la información auditiva en el control motor del sistema del equilibrio en pacientes con implantes cocleares. Anales de la Facultad de Medicina, 6(2), 8-24. https//doi.org/10.25184/ anfamed2019v6n2a10

Teki, S., Grube, M. \& Griffiths, T. (2012). A unified model of time perception accounts for duration-based and beatbased timing mechanisms. Frontiers in Integrative Neuroscience, 5, 1-7. https://doi.org/10.3389/fnint.2011.00 090

Vidranski, T. \& Farkaš, D. (2015). Motor skills in hearing impaired children with or without cochlear implant -A systematic review. Collegium Antropologicum, 39(1), 173-179. https://bit.ly/3kuQrXI

Viljanen, A., Kaprio, J., Pyykko, I., Sorri, M., Pajala, S., Kauppinen, M., Koskenvuo, M. \& Rantanen, T. (2009). Hearing as a Predictor of Falls and Postural Balance in Older Female Twins. The Journals of Gerontology Series A: Biological Sciences and Medical Sciences, 64A(2), 312-317. https://doi.org/10.1093/gerona/gln015 